\title{
Complement C1s inhibition with sutimlimab results in durable response in cold agglutinin disease: CARDINAL study 1-year interim follow-up results
}

Sutimlimab (BIVV009; TNT009) is a humanized monoclonal antibody designed to target $\mathrm{C} 1 \mathrm{~s}$, the $\mathrm{C} 1 \mathrm{complex}$ serine protease responsible for activating the classical complement pathway which triggers hemolysis in cold agglutinin disease (CAD). ${ }^{1}$ Results from the pivotal CARDINAL study showed sutimlimab to be an efficacious and well-tolerated therapy during 26 weeks of treatment among CAD patients. ${ }^{2}$ We present 1-year interim results of the ongoing 2-year CARDINAL extension which show that sutimlimab has a sustained treatment effect in CAD via long-term complement inhibition. Sutimlimab maintained mean hemoglobin levels $\geq 11 \mathrm{~g} / \mathrm{dL}$ with sustained improvement in quality of life; no new safety concerns were identified.

$C A D$ is a rare autoimmune hemolytic anemia characterized by chronic hemolysis mediated entirely by activation of the classical complement pathway. ${ }^{3}$ CAD is a low-grade clonal lymphoproliferative disorder with no underlying overt malignancy or infection. ${ }^{4}$ Clinical manifestations of CAD include classical complement pathway-mediated chronic hemolytic anemia, profound fatigue, acute hemolytic crises, as well as transient, cold-induced, red blood cell agglutination-mediated circulatory symptoms including acrocyanosis. ${ }^{5}$ There are currently no approved therapies for CAD. ${ }^{6}$ Unapproved pharmacological modalities offer varying efficacy and safety, as well as different response rates and response durations. .- $^{6-}$ ${ }^{9}$ Rituximab depletes $\mathrm{B}$ cells and induces a partial response in approximately $50 \%$ of patients, with a median delay of 1.5 months and relapse within 1 year. The addition of cytotoxic agents (bendamustine or fludarabine) to rituximab, although associated with increased response rates, is accompanied by more serious toxicity, including severe neutropenia. ${ }^{8,9}$ Blood transfusions are reserved for acute hemolytic anemia and are only a transient temporizing measure; other treatment is required to restrain CAD-associated hemolysis, to which transfused red blood cells are also subjected. ${ }^{4}$ Thus, an alternative treatment that is non-cytotoxic, rapid, and durable in controlling classical complement-mediated hemolysis and its clinical manifestations in CAD patients is needed.

CARDINAL is a prospective, open-label, single-arm, multicenter trial comprising 16 sites from eight countries (ClinicalTrials.gov identifier: NCT03347396). This two-part study had a 26-week treatment period (part A; completed 11 July, 2019) and an ongoing extension (part B) for 2 years after the last patient had completed part A. Data for the combined study period up to a minimum of 53 weeks of follow-up for all ongoing patients are presented here (data cut: 16 January, 2020). Patients $\geq 18$ years of age with a confirmed diagnosis of CAD, baseline hemoglobin $\leq 10 \mathrm{~g} / \mathrm{dL}$, and a history of recent transfusion ( $\geq 1$ blood transfusions in the preceding 6 months) were enrolled. ${ }^{2}$ Patients were treated with sutimlimab intravenously on days 0 and 7 , followed by biweekly dosing for 2 years. Patients weighing $<75 \mathrm{~kg}$ or $\geq 75$ $\mathrm{kg}$ received sutimlimab $6.5 \mathrm{~g}$ or $7.5 \mathrm{~g}$ fixed dose, respectively. The complete study design and results of part A were reported previously. ${ }^{2}$ Patients had to be vaccinated against encapsulated bacterial pathogens (Neisseria meningitis, including serogroup $B$ meningococcus, Haemophilus influen$z a e$, and Streptococcus pneumoniae) within 5 years before enrollment. Efficacy endpoints for part B included a change from baseline in hemoglobin levels, hemolytic markers (total bilirubin), blood transfusions up to 53 weeks, and quality of life assessed using the Functional Assessment of Chronic Illness Therapy (FACIT)-Fatigue Scale up to 51 weeks (last data recording within the 1-year treatment period). Safety endpoints included incidence of treatmentemergent adverse events (TEAE) and treatment-emergent serious adverse events (TESAE) as well as changes in systemic lupus erythematosus panel parameters up to 53 weeks.

Of 42 patients screened, 24 patients were enrolled and received one or more doses of sutimlimab in part A. ${ }^{2}$ Twentytwo patients (91.7\%) completed part A and entered part B. Most patients were female $(62.5 \%)$ and $\geq 65$ years of age (79.2\%). Patients had reduced mean (standard deviation [SD]) hemoglobin $8.6(1.6) \mathrm{g} / \mathrm{dL}$ and elevated mean (SD) bilirubin $53.3(24.0) \mu \mathrm{mol} / \mathrm{L}$ levels at baseline. ${ }^{2}$ After the first sutimlimab dose, mean (standard error [SE]) hemoglobin levels improved rapidly from baseline by $1.2(0.3) \mathrm{g} / \mathrm{dL}$ within the first week, improved by $2.3(0.3) \mathrm{g} / \mathrm{dL}$ during the third week, and were maintained thereafter at $\geq 11 \mathrm{~g} / \mathrm{dL}$ from week 5 through week 53 (Figure 1). Overall, there was a sustained increase in hemoglobin level of $\geq 2 \mathrm{~g} / \mathrm{dL}$ from week 3 to week 53 , and $55.0 \%$ of patients (11/22) had normalized hemoglobin level ( $\geq 12 \mathrm{~g} / \mathrm{dL}$ ) at week 53 . Mean total bilirubin decreased rapidly by week 1 after sutimlimab, was normalized (<upper limit of normal: $20.5 \mu \mathrm{mol} / \mathrm{L}$ ) by week 3 and remained normalized through to week 53 (Figure 1). Normalization of bilirubin level $(<20.5 \mu \mathrm{mol} / \mathrm{L})$ was achieved in $63.6 \%$ of patients $(14 / 22)$ at week 53 , at which time the mean (SE) change in bilirubin from baseline was a reduction of $-35.3(4.2) \mu \mathrm{mol} / \mathrm{L}$. Seventeen (70.8\%) and 19 (86.4\%) pa- 
tients remained transfusion-free from week 5 to 26 (part A) and week 27 to 53 (part B), respectively (Online Supplementary Table S1).

The baseline mean (SE) FACIT-Fatigue score of 32.5 (2.3) points indicated that quality of life was significantly affected (Figure 1). Following sutimlimab treatment, mean (SE) FACIT-Fatigue score increased to 39.7 (1.8) by week 1 (a 7point improvement) and remained $>40$ from weeks 3 to 51 (last data recording). Overall, there was a mean (SE) improvement of 10.4 (3.5) points by week 26, which was sustained at 11.4 (2.8) points at week 51, consistent with a clinically meaningful improvement ( $\geq 3$-point increase)..$^{10}$ Sutimlimab led to near-complete inhibition of classical complement pathway activity. Mean (SE) classical complement pathway activity declined from $20.0 \%$ (3.4\%) at baseline to $3.0 \%(0.7 \%)$ at week 25 and $3.0 \%(0.7 \%)$ at week 51, along-

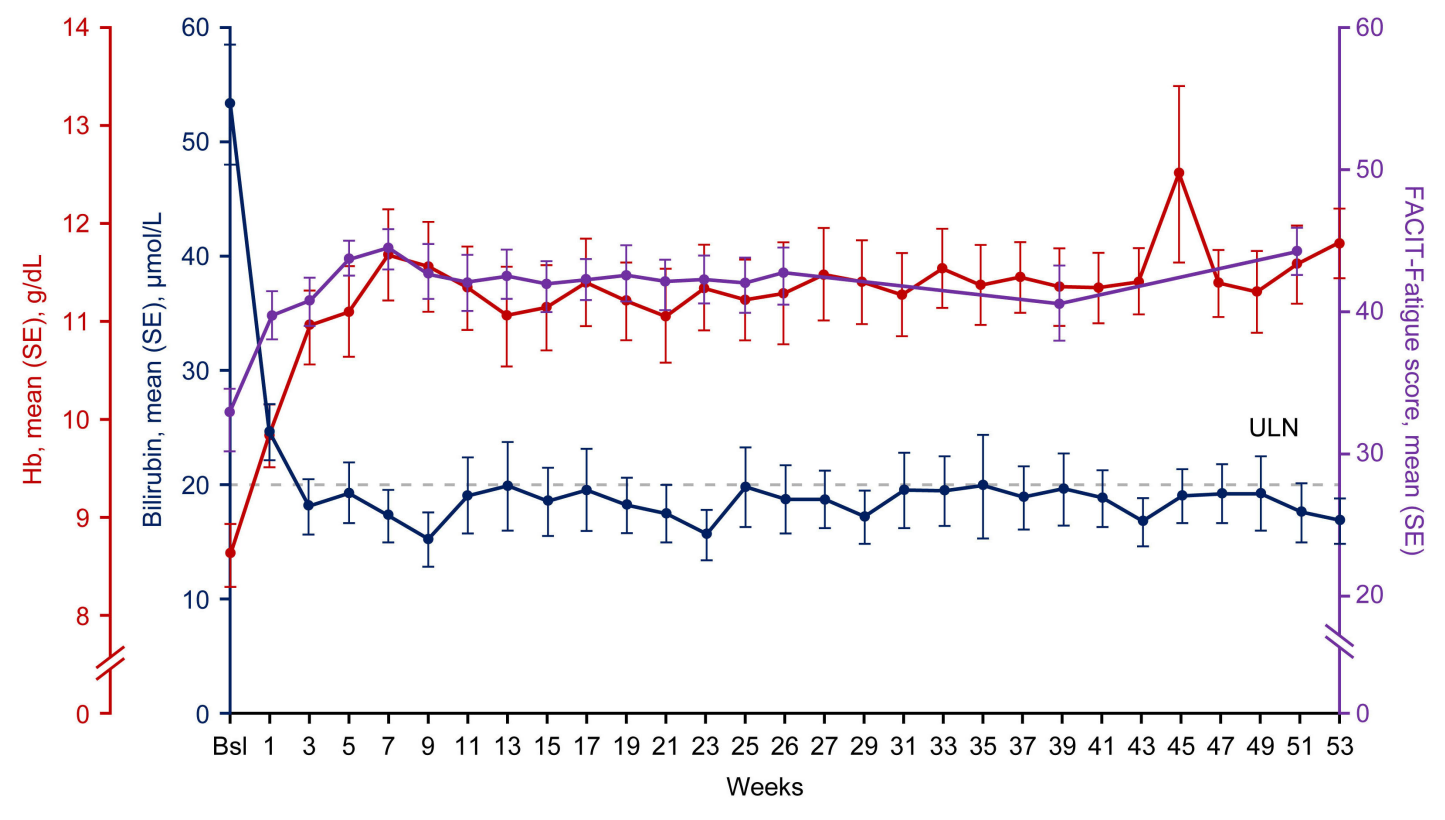

N 2422232322212321222222212121202222212221212222212121201916 N 2120191919172019191919181818171919191919191318161818181619 N 222120201919212121202121202117

20

18

Figure 1. Mean hemoglobin, total bilirubin, and FACIT-Fatigue score from baseline to weeks 51-53 after sutimlimab treatment in patients with cold agglutinin disease (full analysis set). Three patients with a presumed Gilbert's syndrome test result were excluded from the total bilirubin data $(n=21)$. The range of the FACIT-Fatigue score was $0-52$. Patients with a completed questionnaire were included (a questionnaire was completed if $\geq 7$ of 13 items were answered by the patient). FACIT-Fatigue data were not collected at week 53 . The number of patients varied by study visit and by analyte in this interim analysis as the study is ongoing. The data cutoff date was 16 January, 2020. The upper limit of normal (ULN) bilirubin level was defined as $20.5 \mu$ mol/L. Bsl: baseline; FACIT: Functional Assessment of Chronic Illness Therapy; Hb: hemoglobin; SE: standard error.

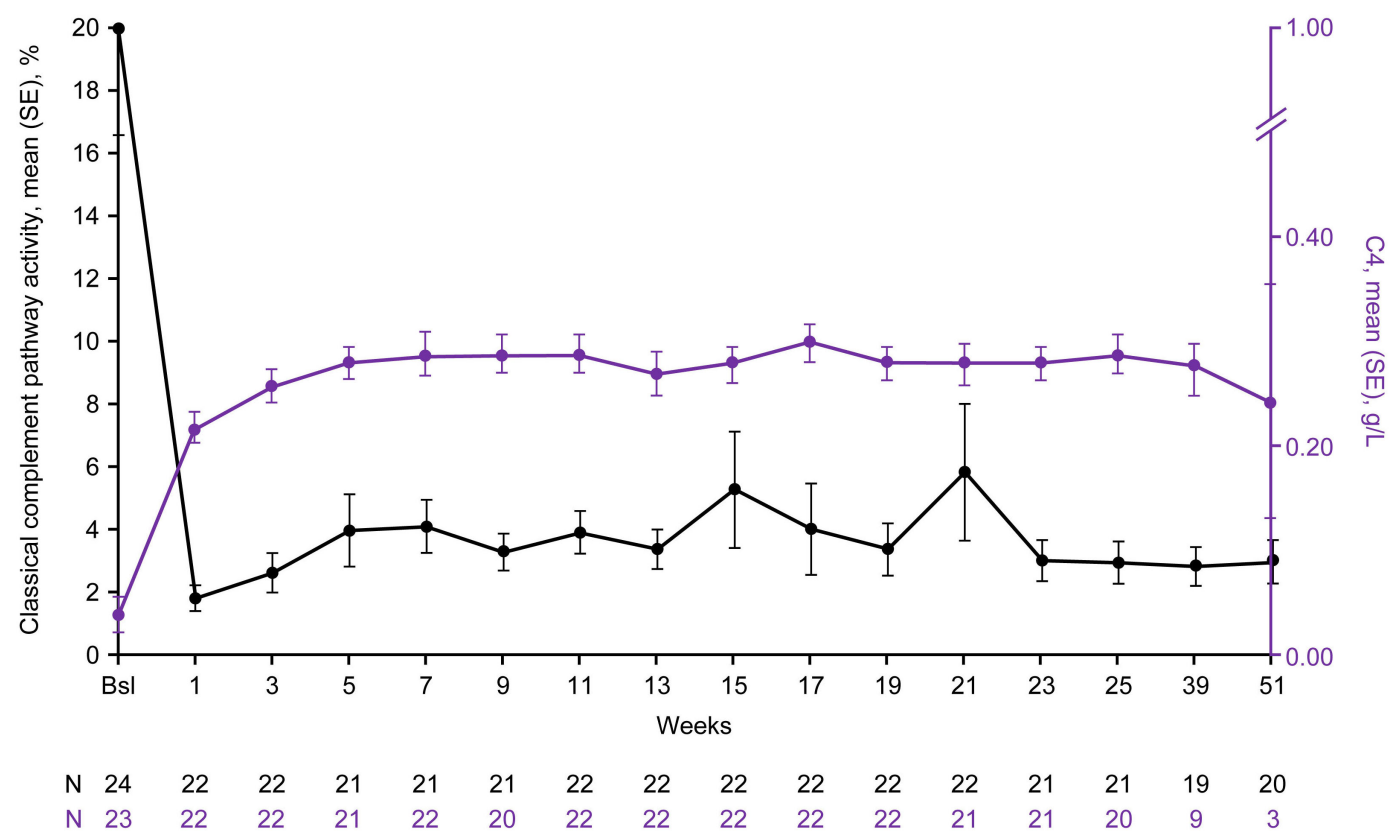

Figure 2. Mean classical complement pathway activity from baseline up to $\mathbf{5 1}$ weeks after sutimlimab treatment in patients with cold agglutinin disease (full analysis set). Classical pathway activity and C4 levels are shown. The number of patients varied by study visit and by analyte in this interim analysis as the study is ongoing. The data cutoff date was 16 January, 2020. Classical pathway activation was determined using an enzyme-linked immunosorbent assay that measures the functional capacity of the classical pathway (Wieslab ${ }^{\circledR}$ classical complement pathway assay; normal range in serum is $69-129 \%$.). The standard international reference range for serum C4 is 0.18 to $0.45 \mathrm{~g} / \mathrm{L}$. Pharmacodynamic assessments were performed at 3-month intervals during the first year of treatment in part B and then at 6-month intervals. Bsl: baseline; SE: standard error. 
side normalization of complement $\mathrm{C} 4$ levels (defined as <normal range: $0.18-0.45 \mathrm{~g} / \mathrm{L}$ ). Mean (SE) total C4 level at baseline was $0.04(0.02) \mathrm{g} / \mathrm{L}$, at week 25 it was $0.29(0.02)$ $\mathrm{g} / \mathrm{L}$, and at week 51 it was 0.24 (0.11) g/L (Figure 2). This coincided with improvements seen in hemoglobin, bilirubin, and FACIT-Fatigue score.

From baseline to week 53 , all 24 patients experienced $\geq 1$ TEAE; nine patients (37.5\%) had $\geq 1$ TEAE related to sutimlimab (Table 1), with the most common being acrocyanosis/cyanosis and infusion-related reaction (two patients each [8.3\%]). Of the $281 \mathrm{TEAE}$, the most common were diarrhea and nasopharyngitis (20.8\% each), and anemia, nausea, and hypertension (16.7\% each). In part B, one event of device-related thrombosis was reported and considered by the investigator related to the indwelling catheter and not related to sutimlimab. Overall, 57 infections were reported as TEAE in all 24 patients and nine TEAE grade $\geq 3$ infections were reported in six patients (25.0\%). Four TEAE (grade 1 or 2) suggestive of potential hypersensitivity to sutimlimab were reported in three patients; all resolved and

Table 1. Summary of treatment-emergent adverse events (safety analysis set).

\begin{tabular}{|c|c|}
\hline & Parts $A$ and $B$ Total $(N=24)$ \\
\hline $\begin{array}{l}\text { TEAE, N } \\
\text { Patients with } \geq 1 \text { TEAE, N (\%) } \\
\text { Most common TEAE (>10\%) } \\
\text { Diarrhea, N (\%) } \\
\text { Nasopharyngitis, N (\%) } \\
\text { Anemia, N (\%) } \\
\text { Hypertension, N (\%) } \\
\text { Nausea, N (\%) } \\
\text { Confusional state, N (\%) } \\
\text { Constipation, N (\%) } \\
\text { Contusion, N (\%) } \\
\text { Cough, N (\%) } \\
\text { Cyanosis, N (\%) } \\
\text { Cystitis, N (\%) } \\
\text { Dizziness, N (\%) } \\
\text { Fatigue, N (\%) } \\
\text { Gastroenteritis, N (\%) } \\
\text { Hemorrhoids, N (\%) } \\
\text { Upper abdominal pain, N (\%) } \\
\text { Upper respiratory tract infection, N (\%) }\end{array}$ & $\begin{array}{c}281 \\
24(100.0) \\
5(20.8) \\
5(20.8) \\
4(16.7) \\
4(16.7) \\
4(16.7) \\
3(12.5) \\
3(12.5) \\
3(12.5) \\
3(12.5) \\
3(12.5) \\
3(12.5) \\
3(12.5) \\
3(12.5) \\
3(12.5) \\
3(12.5) \\
3(12.5) \\
3(12.5)\end{array}$ \\
\hline $\begin{array}{l}\text { Patients with } \geq 1 \text { related TEAE, }{ }^{*} \mathrm{~N}(\%) \\
\text { Total number of related TEAE, } \mathrm{N} \\
\text { Most common related TEAE (>1 patient) } \\
\text { Acrocyanosis/cyanosis, } \mathrm{N}(\%) \\
\text { Infusion-related reaction, } \mathrm{N}(\%)\end{array}$ & $\begin{array}{c}9(37.5) \\
25^{\dagger} \\
2(8.3) \\
2(8.3)\end{array}$ \\
\hline Patients with $\geq 1$ TEAE grade 3 or higher, $N(\%)$ & $14(58.3)$ \\
\hline $\begin{array}{l}\text { Patients with } \geq 1 \text { TEAE infection grade } 3 \text { or higher, } \mathrm{N}(\%) \\
\text { Total number of TEAE infections, } \mathrm{N} \\
\text { Total number of TESAE infections, } \mathrm{N} \\
\text { Total number of TEAE infection grade } 3 \text { or higher, } \mathrm{N}\end{array}$ & $\begin{array}{c}6(25.0) \\
57 \\
9 \\
9\end{array}$ \\
\hline $\begin{array}{l}\text { TESAE, } N \\
\text { Patients with } \geq 1 \text { TESAE, } N(\%) \\
\text { Patients with } \geq 1 \text { related TESAE, }{ }^{*} \mathrm{~N}(\%)\end{array}$ & $\begin{aligned} & 30 \\
12 & (50.0) \\
1 & (4.2)\end{aligned}$ \\
\hline $\begin{array}{l}\text { Total number of TEAE thromboembolic events, } N \\
\text { Patients with } \geq 1 \text { TESAE thromboembolic event, } N(\%)\end{array}$ & $\begin{array}{c}1 \neq \\
1(4.2)\end{array}$ \\
\hline Patients who discontinued treatment and/or study owing to a TEAE, N (\%) & $2(8.3)^{\S}$ \\
\hline Deaths, N (\%) & $1(4.2)^{\|}$ \\
\hline
\end{tabular}

Data cutoff date was 16 January; 2020. ${ }^{2}$ Adverse events with missing causality assessment were included in the related treatment-emergent adverse events (TEAE)/treatment-emergent serious adverse events (TESAE); adverse events with investigator causality assessment of "possible" or "probable" were considered related. 'Events comprised pain in both hands/legs related to acrocyanosis/cyanosis, dysphagia, application-site hemorrhage, fatigue, peripheral edema, temperature intolerance, cystitis, upper respiratory tract infection, viral infection, contusion, infusion-related reaction, hypertension, arthralgia, tendonitis, dyspnea, rhinorrhea, and erythema. *Deep vein thrombosis in the right arm due to an indwelling catheter. ${ }^{5}$ One treatment/study discontinuation due to the patient's death (as per footnote"); one treatment/study discontinuation due to multiple non-serious TEAE, including acrocyanosis due to cold agglutinin disease and dysphagia, which were both assessed as related to sutimlimab. A third patient discontinued treatment/study because of a pretreatment serious adverse event of polymyalgia rheumatica. "One patient died of progressive carcinoma (unrelated to study treatment). 
did not recur, and none led to study discontinuation. Three patients experienced TEAE of acrocyanosis/cyanosis, of whom one had a history of Raynaud's phenomenon. One patient had a TESAE of disabling circulatory symptoms with livid foot discoloration, not considered related to sutimlimab treatment by the investigator. No patients developed systemic lupus erythematosus, nor were there any TEAE consistent with the development of other autoimmune diseases. Thirty TESAE were reported in $12 \mathrm{pa}-$ tients (50\%). Serious infections with encapsulated bacteria (Streptococcus pyogenes, Streptococcus pneumoniae, Escherichia coli, and Staphylococcus species) were reported, but no meningococcal infections or TEAE of meningitis were identified. No patient discontinued the study due to an infection. Two patients discontinued the treatment and/or study during the 53 weeks for reasons unrelated to study treatment; post-treatment hemolytic parameters were not collected for these patients. One treatment/study discontinuation occurred due to multiple non-serious TEAE, including acrocyanosis and dysphagia; 9 weeks after treatment, hemoglobin levels remained stable, however all other hemolytic parameters assessed were not within normal range; FACIT-Fatigue score was 7 points below baseline.

The 1-year interim CARDINAL study follow-up further demonstrates that continued classical complement pathway inhibition with sutimlimab results in sustained remission of hemolysis in CAD patients, with durably increased hemoglobin levels, normalized bilirubin levels, and improved FACIT-Fatigue scores. After sutimlimab treatment, the number of blood transfusions decreased with time and most patients remained transfusion-free from weeks 5 to 53 , which translates into reduced patient burden and utilization of healthcare resource.

Patients with CAD in this study had comparable baseline mean FACIT-Fatigue scores to patients with other serious chronic conditions, including rheumatoid arthritis, advanced cancer-related anemia, and paroxysmal nocturnal hemoglobinuria. ${ }^{1-14}$ Within 1 week of sutimlimab treatment, there was a mean increase of 7.24 points from baseline in the FACIT-Fatigue score, which was improved further and sustained throughout the follow-up period, indicating improved quality of life. These improvements coincided with reduced classical complement pathway activity. In addition to anemia driven by hemolytic activity, inflammation associated with classical complement pathway activation may be a key driver of fatigue in patients with CAD. ${ }^{15}$ In summary, 1-year interim results of the ongoing CARDINAL study demonstrate continued inhibition of the classical complement pathway at $\mathrm{C} 1 \mathrm{~s}$ with sutimlimab and sustained treatment effects in CAD. Sutimlimab demonstrated an acceptable safety profile at 1 year; no new safety signals were identified. No TEAE suggestive of serious hypersensitivity or anaphylactic reactions associated with sutimlimab were identified. Other than one devicerelated thrombosis, no other vascular thromboembolic TEAE were reported. These data reinforce the positive riskbenefit profile of sutimlimab as an effective long-term therapy with an acceptable safety profile for management of patients with chronic CAD, particularly with symptoms influenced predominantly by activation of the classical complement pathway (e.g., chronic hemolysis, anemia, and fatigue).

\section{Authors}

Alexander Röth, ${ }^{1}$ Wilma Barcellini, ${ }^{2}$ Shirley D'Sa, ${ }^{3}$ Yoshitaka Miyakawa, ${ }^{4}$ Catherine M. Broome, ${ }^{5}$ Marc Michel, ${ }^{6}$ David J. Kuter, ${ }^{7}$ Bernd Jilma, ${ }^{8}$ Tor Henrik Anderson Tvedt, ${ }^{9}$ Ilene C. Weitz, ${ }^{10}$ Parija Patel, ${ }^{11}$ Xiaoyu Jiang, ${ }^{11}$ Caroline Reuter, ${ }^{11}$ Jun Su, ${ }^{11}$ Frank Shafer, ${ }^{11}$ Michelle Lee ${ }^{11}$ and Sigbjørn Berentsen ${ }^{12}$

${ }^{1}$ Department of Hematology and Stem Cell Transplantation, West German Cancer Center, University Hospital Essen, University of Duisburg-Essen, Essen, Germany; 'Fondazione IRCCS Ca' Granda Ospedale Maggiore Policlinico, Milan, Italy; ${ }^{3} \mathrm{UCLH}$ Centre for Waldenström's Macroglobulinemia and Related Conditions, University College London Hospitals NHS Foundation Trust, London, UK; ${ }^{4}$ Thrombosis and Hemostasis Center, Saitama Medical University Hospital, Saitama, Japan; ${ }^{5}$ Division of Hematology, MedStar Georgetown University Hospital, Washington, DC, USA; ${ }^{6}$ Henri-Mondor University Hospital, Assistance Publique-Hôpitaux de Paris, UPEC, Créteil, France; ${ }^{7}$ Division of Hematology, Massachusetts General Hospital, Harvard Medical School, Boston, MA, USA; ${ }^{8}$ Department of Clinical Pharmacology, Medical University of Vienna, Vienna, Austria; 9Section for Hematology, Department of Medicine, Haukeland University Hospital, Bergen, Norway; ${ }^{10} \mathrm{Keck}$ School of Medicine of USC, Los Angeles, CA, USA; ${ }^{11}$ Sanofi, Cambridge, MA, USA and ${ }^{12}$ Department of Research and Innovation, Haugesund Hospital, Haugesund, Norway

Correspondence:

ALEXANDER RÖTH - alexander.roeth@uk-essen.de

https://doi.org/10.3324/haematol.2021.279812

Received: August 19, 2021.

Accepted: February 9, 2022.

Prepublished: February 17, 2022.

Data were first presented at the $62^{\text {nd }}$ American Society of Hematology Virtual Annual Congress, 5-8 December, 2020.

\section{Disclosures}

AR has received research support from Roche, received honoraria, and provided consultancy to Alexion Pharmaceuticals, Inc., Apellis Pharmaceuticals, Novartis, Roche, Kira, Bioverativ, a Sanofi company, Sanofi and Sobi. SD has received grant funding, honoraria, and/or speaker's fees from Janssen, BeiGene, and Sanofi. YM has provided 
consultancy for Bioverativ and Sanofi. WB has received research support from Alexion Pharmaceuticals, Inc., and Novartis; participated in advisory boards for Agios, Alexion Pharmaceuticals, Inc., Bioverativ, and Incyte; and has been an invited speaker for Alexion Pharmaceuticals, Inc., and Novartis. CMB has received honoraria and/or research funding from Alexion Pharmaceuticals, Inc., Bioverativ, Cellphire, Incyte, Rigel, and Sanofi Genzyme. MM has provided consultancy to Alexion Pharmaceuticals, Inc., Rigel, and Bioverativ. DJK has provided consultancy to Actelion (Syntimmune), Agios, Alnylam, Amgen, Argenx, Bristol-Myers Squibb, Caremark, Daiichi Sankyo, Dova, Kyowa Kirin, Merck Sharp \& Dohme, Momenta, Novartis, Pfizer, Platelet Disorder Support Association, Principia, Protalex, Protalix Biotherapeutics, Rigel, Sanofi, Shionogi, Shire, Takeda (Bioverativ), UCB, Up-To-Date, and Zafgen; and received research funding from Actelion (Syntimmune), Agios, Alnylam, Amgen, Argenx, Bristol-Myers Squibb, Kezar Life Sciences, Inc., Principia, Protalex, Rigel, and Takeda (Bioverativ). BJ has received reimbursement for travel costs for scientific presentations and consultancy to True North Therapeutics, Bioverativ and Sanofi. THAT has participated in advisory boards for Alexion Pharmaceuticals, Inc., Novartis, and Ablynx. ICW has received honoraria from Alexion Pharmaceuticals Inc. and Sanofi. XJ, ML, and FS are employees of Sanofi and may hold shares and/or stock options in the company. PP, JS, and CR were employees of Sanofi at the time of the study. SB has received research funding from Mundipharma; lecture honoraria from Apellis Pharmaceuticals, Alexion Pharmaceuticals, Inc., Bioverativ, Janssen-Cilag, and Sanofi; and provided consultancy to Apellis Pharmaceuticals, Bioverativ, Sanofi, and True North Therapeutics.

\section{Contributions}

AR, FS, PP, ML, JS, and CR analyzed/reviewed data. XJ reviewed the results of the statistical analysis. All authors had access to primary clinical trial data, had full editorial control of the manuscript, and provided their final approval of all content.

\section{Acknowledgments}

We would like to thank the investigators, healthcare providers, research staff, and patients who participated in the CARDINAL study. The authors are grateful for the assistance provided by Jennifer Wang and Katarina Kralova of Sanofi.

\section{Funding}

This study was funded by Sanofi (Waltham, MA, USA). Medical writing and editing support were provided by Rhutika Dessai and Santo D’Angelo of Fishawack Communications Ltd., part of Fishawack Health, and by Lisa Buttle and Sally Ratcliffe of Lucid Group, and funded by Sanofi.

\section{Data-sharing statement}

Qualified researchers may request access to patient-level data and related study documents such as the clinical study report, study protocol (with amendments), statistical analysis plan, and dataset specifications. Of note, patient-level data will be anonymized, and study documents will be redacted in order to protect the privacy of trial participants. Further information related to Sanofi's data sharing criteria, eligible studies, and process for requesting access can be found at: https://www.clinicalstudydatarequest.com/.

\section{References}

1. Bartko J, Schoergenhofer C, Schwameis M, et al. A randomized, first-in-human, healthy volunteer trial of sutimlimab, a humanized antibody for the specific inhibition of the classical complement pathway. Clin Pharmacol Ther. 2018;104(4):655-663.

2. Röth A, Barcellini W, D'Sa S, et al. Sutimlimab in cold agglutinin disease. N Engl J Med. 2021;384(14):1323-1334.

3. Berentsen S, Ulvestad E, Langholm R, et al. Primary chronic cold agglutinin disease: a population based clinical study of 86 patients. Haematologica. 2006;91(4):460-466.

4. Jäger $\mathrm{U}$, Barcellini W, Broome CM, et al. Diagnosis and treatment of autoimmune hemolytic anemia in adults: recommendations from the First International Consensus Meeting. Blood Rev. 2020;41:100648.

5. Berentsen S. Cold agglutinin disease. Hematology Am Soc Hematol Educ Program. 2016;2016(1):226-231.

6. Jia MN, Qiu Y, Wu YY, et al. Rituximab-containing therapy for cold agglutinin disease: a retrospective study of 16 patients. Sci Rep. 2020;10(1):12694.

7. Röth A, Bommer M, Hüttmann A, et al. Eculizumab in cold agglutinin disease (DECADE): an open-label, prospective, bicentric, nonrandomized phase 2 trial. Blood Adv. 2018;2(19):2543-2549.

8. Berentsen S, Randen U, Vågan AM, et al. High response rate and durable remissions following fludarabine and rituximab combination therapy for chronic cold agglutinin disease. Blood. 2010;116(17):3180-3184.

9. Berentsen S, Randen U, Oksman M, et al. Bendamustine plus rituximab for chronic cold agglutinin disease: results of a Nordic prospective multicenter trial. Blood. 2017;130(4):537-541.

10. Nordin A, Taft C, Lundgren-Nilsson A, Dencker A. Minimal important differences for fatigue patient reported outcome measures-a systematic review. BMC Med Res Methodol. 2016;16:62.

11. Cella D, Yount S, Sorensen M, Chartash E, Sengupta N, Grober J. Validation of the Functional Assessment of Chronic Illness Therapy Fatigue Scale relative to other instrumentation in patients with rheumatoid arthritis. J Rheumatol. 2005;32(5):811-819.

12. Cella D, Eton DT, Lai JS, Peterman AH, Merkel DE. Combining anchor and distribution-based methods to derive minimal clinically important differences on the Functional Assessment of Cancer Therapy (FACT) anemia and fatigue scales. J Pain Symptom Manage. 2002;24(6):547-561.

13. Escalante CP, Chisolm S, Song J, et al. Fatigue, symptom burden, and health-related quality of life in patients with myelodysplastic syndrome, aplastic anemia, and paroxysmal nocturnal hemoglobinuria. Cancer Med. 2019;8(2):543-553.

14. Schrezenmeier H, Röth A, Araten DJ, et al. Baseline clinical characteristics and disease burden in patients with paroxysmal nocturnal hemoglobinuria (PNH): updated analysis from the International PNH Registry. Ann Hematol. 2020;99(7):1505-1514.

15. Weitz IC, Ueda Y, Shafer F, et al. Inflammation and fatigue in patients with cold agglutinin disease (CAD): analysis from the phase 3 Cardinal study. Blood. 2020;136(suppl 1):7-8, abstract 759. 